\title{
Recurrent Vulvovaginal Candidiasis
}

\section{Cyntya Sari Sovianti ${ }^{*}$, Mutia Devi ${ }^{1}$}

${ }^{1}$ Department of Dermatology and Venereology Medical Faculty of Sriwijaya University

\section{A R T I C L E I N F O Keywords:}

Recurrent Vulvovaginal Candidiasis Predisposing Factors

Pathogenesis

Management

*Corresponding author:

Cyntya Sari Sovianti

E-mail address:

cyntya@yahoo.com

All authors have reviewed and approved the final version of the manuscript.
A B S T R A C T

Vulvovaginal candidiasis is a common fungal infection caused by Candida $\mathrm{Sp}$, especially Candida albicans. Recurrent vulvovaginal candidiasis was defined as the occurrence of four or more episodes of vulvovaginal candidiasis in 12 months period. As many as $9 \%$ of women from various populations have recurrent vulvovaginal candidiasis. Vulvovaginal candidiasis affects the quality of life, mental health, and sexual activity. There are many predisposing factors that caused recurrent vulvovaginal candidiasis, such as genetics, host, habit, idiopathic and non-albican candida microbes. Management of recurrent vulvovaginal candidiasis includes elimination of predisposing factors; mycological culture diagnosis and identification of specific Candida species; followed by microbiological examination to confirm the sensitivity of the azole group to Candida sp. Further, oral, or topical therapy should be continued until the patient is asymptomatic and culture-negative. Patients should receive induction therapy followed by maintenance suppressive therapy for six months.

\section{https://doi.org/10.32539/bsm.v5i5.280}

\section{Introduction}

Candidiasis is an infection with various clinical manifestations caused by candida, particularly Candida albicans and other yeasts of the genus Candida. Candidiasis that affects the vulva and vagina is called vulvovaginal candidiasis (CVV). Recurrent vulvovaginal candidiasis is defined as vulvovaginal candidiasis that recurs 4 or more times a year. ${ }^{1}$

Vulvovaginal candidiasis is the second most common form of vaginal infection after bacterial vaginosis, vulvovaginal candidiasis affects $75-80 \%$ of women at least once in a lifetime. The incidence of recurrent vulvovaginal candidiasis in women is $9 \%$. Moreover, worldwide, the prevalence and epidemiological data on recurrent vulvovaginal candidiasis are sparse and inaccurate as they are generally self-reported and diagnosed by local general practitioners. Denning et al. Conducted a systematic review of epidemiological studies from 1985-2016 of 6.000 online surveys in five western European countries and the United States which found the prevalence of recurrent vulvovaginal candidiasis is 3871 cases per 100,000 women, with the highest frequency are $9 \%$ at $25-34$ years women. ${ }^{2}$

Vulvovaginal candidiasis is characterized by a pruritus or irritation of the vulva and vaginal discharge. Vaginal discharge is mucoid or liquid with granules called cottage cheeses. Complaints of dyspareunia and painful urinating can also be found. ${ }^{1}$ Recurrent vulvovaginal candidiasis affects the quality of life, mental health and sexual activity of women. 3 The purpose of this literature review is to recall the risk factors, pathogenesis, diagnosis and management of recurrent vulvovaginal candidiasis. 


\section{Etiology}

Epidemiological studies in America, Europe and Australia found that Candida albicans is the species most frequently found as a cause of vulvovaginal candidiasis, which is $75-90 \%$, while non-Candida albicans includes Candida glabrata is $10-20 \%$ of cases, followed by Candida parapsilosis, Candida tropicalis, Candida krusei and Candida africana. Studies in Tunisia, Nigeria, Middle Eastern and Asian countries reported that Candida glabrata is the most common species, which is $30-50 \% .^{2}$ Non-albican candida infection is believed to be one of the causes of recurrent vulvovaginal candidiasis, it is caused by resistant nonalbicans candida against the azole agents which is the main therapy for vulvovaginal candidiasis. ${ }^{2}$

\section{Predisposition factors}

Recurrent vulvovaginal candidiasis can occur by a variety of mechanisms or idiopathic (Figure 1). Predisposing factors for vaginal colonization can differ from those that facilitate the transformation of asymptomatic vaginitis to symptomatic. 4

\section{Genetic}

The prevalence of vulvovaginal candidiasis is influenced by genetic factors. A study found an increased prevalence of vulvovaginal candidiasis in African-American women. ${ }^{4}$ The single nucleotide polymorphism (SNP) at exon 1 codon 54 in the mannose-binding lectin 2 (MBL2) gene was frequently seen with recurrent vulvovaginal candidiasis. ${ }^{2}$ The risk of recurrent vulvovaginal candidiasis occurring in the heterogeneity of the MBL2B allele is increase.3.6 Further, in vivo polymorphism studies found an association between the mannose-binding lectin (MBL) and the occurrence of vaginal candida colonization or vaginitis. In polymorphism, there is a lack of MBL levels and disruption of the toll-like receptors (TLR) function which functions to recognize components in the candida cell walls. Both of these lead to reduced vaginal defense mechanisms against candida. ${ }^{5}$ Nonsynonymous polymorphisms at toll-like receptor 2 (Pro631His, rs5743704) were found to be threefold in women with recurrent vulvovaginal candidiasis. 3,7 In a study involving 270 of recurrent vulvovaginal candidiasis patients and 583 healthy controls, the 12/9 genotypes of NLRP3 gene were more in cases than controls. ${ }^{3.8}$

\section{Pregnancy}

During pregnancy the prevalence of vaginal candida colonization and symptomatic vaginitis increases. ${ }^{9}$ The increased risk of candidiasis vulvovaginitis and asymptomatic colonization in pregnancy is likely due to factors associated with pregnancy, such as decreased cell-mediated immunity, increased estrogen levels, and increased mucosal glycogen production in vagina. Moreover, the Increased level of reproductive hormones resulting in the increased level of vaginal mucosal glycogen levels which is a carbon source for candida. 9 Further, estrogen facilitates the attachment of yeast to epithelial cells of vaginal mucosa. In addition, estrogen stimulates hyphae formation and elaboration of enzymes, such as aspartyl proteinase and secreted phospholipase. These virulence factors can further enhance colonization. 10

As gestational age increases, reproductive hormone levels change dramatically and higher than at other times. The interactions between hormones and the immune system are complex and multifactorial. Take for example a High level of estradiol enhance several aspects of natural and cell-mediated immunity (Th2 response) and humoral adaptive immune responses. Progesterone also changes the balance between Th1 and Th2 responses. ${ }^{10}$

This aspect of natural immunity increases during pregnancy, especially during the second and third trimesters, contributing to lower the down-regulation of the genital tract immune system. In addition, the levels of some cytokines are increased which leads to the recruitment or activity of phagocytes. In contrast, CD3 and $\mathrm{T}$ lymphocyte counts decrease during pregnancy, as do Th1 and Th2 responses to antigenic lymphocytic stimulation. 10

More recent theories suggest a shift from Th1 to Th2 immunity. Th2 cells stimulate the B lymphocyte 
response, increase antibody production, and suppress the $\mathrm{T}$ lymphocyte response, which decreases the cellmediated immunity that is responsible for altered response to infection during pregnancy. The Th2 profile, characterized by elevated levels of IL-4, IL-5, and IL-13, is considered to contribute to recurrent candidiasis vulvovaginitis. 10

\section{Contraception}

Several studies have shown an increased in vaginal candida colonization with the use of oral contraceptives with high estrogen levels. Other studies have shown that women who use oral contraceptives with low estrogen levels do not show an increase in vulvovaginal candidiasis. Moreover, studies have reported an increased number of yeasts in the use of intrauterine devices, diaphragms, condoms and sponges. ${ }^{9}$

\section{Diabetes mellitus}

Vaginal candida colonization is more common in women with diabetes mellitus. ${ }^{9}$ A cross-sectional study in Brazil reported that women with diabetes mellitus had a higher vaginal candida colonization, as much as $18.8 \%$ when compared with those without diabetes mellitus by 1\%. Symptomatic vaginitis (vulvovaginal candidiasis and recurrent vulvovaginal candidiasis) in women with diabetes mellitus was found in $66.6 \%$, while vaginal colonization was found in 33.3\%.2 Women with noninsulin dependent diabetes mellitus tend to experience Candida glabrata colonization. Although uncontrolled diabetes is undoubtedly a predisposition to symptomatic vaginitis, controlled diabetes also does not avoid the increased prevalence of vulvovaginal candidiasis. Examination of glucose tolerance test is a routine examination performed in all women with recurrent vulvovaginal candidiasis. Blood sugar levels are believed to be associated with exacerbation of symptomatic vaginitis. 9

High serum glucose levels inhibit monocyte, granulocyte, and neutrophil function as well as reducing chemotaxis, phagocytosis, and the elimination of pathogens. Glucose is also a source of nutrition for candida. Further, research has shown that there is a relationship between enzyme activity and pathogenicity of candida. Enzymatic activity is also influenced by blood sugar levels. The higher the blood sugar level, the higher the enzymatic activity. Enzymes that affect the virulence of candida include Secretory aspartyl proteinases (SAP) and phospholipase. Increased levels of glycogen will lower vaginal $\mathrm{pH}$ and facilitate vulvovaginal candidiasis. ${ }^{11}$

\section{Antibiotics}

The onset of recurrent vulvovaginal candidiasis is influenced by the use of antibiotics. Vulvovaginal candidiasis occurs in $28-33 \%$ after antibiotic use. The use of antibiotics eliminates the normal vaginal flora, cause to more candida growth. Lactobacillus spp is one of normal vaginal flora will creates a state of resistance to candida colonization, keeps the yeast in count low and prevents invasion of the superficial mucosa.

\section{Habit}

The increased incidence of vulvovaginal candidiasis is influenced by sexual activity and the use of hormone replacement therapy. Transmission of Candida sp can occur during sexual activity. Several studies have found an association between the frequency sexual intercourse and the occurrence of vulvovaginal candidiasis. Other studies have shown a relationship between vulvovaginal candidiasis and orogenital receptive sex. The use of well-ventilated clothing and cotton underwear can prevent infection. Chemical contact, atopic and hypersensitivity reactions will affect the vaginal environment and facilitate the transformation of asymptomatic to symptomatic colonization. 9

\section{Bacterial vaginosis}

Bacterial vaginosis is a syndrome when the vaginal ecosystem is changed from Lactobacillus sp, that normally produce hydrogen peroxide $\left(\mathrm{H}_{2} \mathrm{O}_{2}\right)$ to anaerobic bacterial such as Prevotella sp, Mobiluncus $\mathrm{sp}$, Gardenela vaginalis and Mycoplasma homini. This change will cause the increase of vaginal $\mathrm{pH}$. 
In the vagina, microbes have a mutualistic relationship with the host and act as a defense against pathogenic organisms. ${ }^{12}$ Lactobacillus sp is the main source of lactic acid. The functions of physiological concentration of lactic acid are to acidify vaginal secretions (to a pH level <4), increase $\mathrm{H}_{2} \mathrm{O}_{2}$ and bacteriocin activity, and inhibit opportunistic infections. Glycogen from exfoliated and lysed epithelial cells is catabolized by a-amylase present in the vaginal lumen into smaller polymers which are then metabolized to lactic acid by Lactobacillus sp. Lactic acid and cytolysin produced by Lactobacillus $\mathrm{sp}$ stimulate the dissolution of epithelial cells by lysis and increase the availability of glycogen. Lactic acid acidifies the vaginal environment which supports the proliferation of Lactobacillus sp and inhibits the growth of infection-related organisms. This is reinforced by Lactobacilli through the production of $\mathrm{H}_{2} \mathrm{O}_{2}$, bacteriocins and biosurfactants, as well as inhibition of physical attachment of pathogens to the epithelium with competitive exclusion and stimulates ingestion and degradation of infected epithelial cells (autophagy). In addition, there is the concurrent production of mucin, immunoglobulins (secretion of IgA and IgG), secretory leucocyte protease inhibitors (SLPI), neutrophil gelatinase-associated lipocalin (NGAL), $\beta$ defensin, and other antimicrobial proteins, which together provide a defense against infection. If the number of Lactobacillus $\mathrm{sp}$ is reduced, pathogenic organisms can potentially grow and cause various pathological conditions. 13

\section{Atopic}

A person with atopic dermatitis (AD) is prone to various infections including fungal infections. In $\mathrm{AD}$, there is impaired function of the skin barrier, the microorganisms present in the skin can penetrate easily and interact with the immune system. ${ }^{14}$ In women with recurrent vulvovaginal candidiasis there is a decrease in delayed-type hypersensitivity reactions to the Candida albicans antigen. In vitro studies have shown impaired Th1 response resulting in insufficient production of interferon $\gamma$ specific for Candida albicans in women with recurrent vulvovaginal candidiasis. ${ }^{14,15,16}$

\section{Steroids}

Steroids have been used widely as antiinflammatory and immunosuppressive. Long-term steroid use is associated with an increased incidence of vulvovaginal candidiasis. Decreased immunity of women who use long-term steroids causes infection with opportunistic microorganisms, one of which is candida infection. The use of steroids will result in increased blood glucose levels which will affect the vaginal environment and increase the proliferation of yeast cells. 17

\section{Human immunodeficiency virus (HIV)}

Human immunodeficiency virus infects CD4+ $\mathrm{T}$ lymphocytes, causing an immunosuppressive state. The spectrum of candidiasis in HIV patients is very broad, it can affect the mucosa, skin, nails, even disseminated candidiasis and candidemia. Mucosal candidiasis, whether oral, esophageal or vaginal infection is a common form of HIV patients. Vulvovaginal candidiasis is the most common clinical condition in women with HIV. Infection tends to be persistent, especially in patients with low CD4 cells and high viral loads. As with other opportunistic infections, the presence of highly active antiretroviral therapy (HAART) causes a decreased incidence of candidiasis. 18

\section{Vulvar dermatosis}

Vulvar dermatosis is an inflammatory skin condition that is restricted to the vulva but can also involve the extragenital area.19 Vulvar dermatosis which is frequently co-infected with candida includes contact dermatitis and atopic dermatitis. Disruption of the skin barrier results in the formation of a portal of entry for bacteria, fungi or viruses. Defensin is a component of the natural immune system that acts as antibacterial, antifungal and antiviral. Cytokines that play a role in contact and atopic dermatitis including interleukins 4,10 and 13 will decrease defensin 
regulation so that it will facilitate infection by microbes. 20

\section{Pathogenesis}

There are two mechanisms of recurrent vulvovaginal candidiasis. The first mechanism is transformation of asymptomatic to become symptomatic fungal colonies. The other mechanism is recurrent infection later become chronic infection. ${ }^{9}$ Blastospores of Candida migrate from lower gastrointestinal tract to vaginal lumen through perianal area. 5,9 Colonization of yeast is usually small and asymptomatic. Estrogens will influence the yeast colony to increase the size and to stimulate transition between yeast to become hyphae. Later, this colony will become pathogen and symptomatic. In this condition, in female with predisposition factors such as genetic, biologic and habit, the vulvovaginal candidiasis will become chronic or recurrent. 5

\section{Virulence factors in candida species}

Candida is eucaryotic microorganism that has 2 different phases of life, namely commensal flora and opportunistic pathogen in human and mammals. This characteristic is accommodated by its ability to change their morphology from round-oval yeast (Y) and become mycelium hyphae $(\mathrm{H})$, called dysmorphic transition. Study concluded that morphology of yeast is related to commensalism while morphology of hyphae is related to pathogenicity. Yeast-shaped candida is commonly found in gastrointestinal tract and vagina of more than $50 \%$ asymptomatic female, while hyphae is always found from tissue that been infiltrated by candida such as vulvovaginal candidiasis and recurrent vulvovaginal candidiasis. 21

Yeasts, the commensal flora, are tolerated by host and retained in small numbers in vagina by various mechanisms that inhibit the transition to hyphae. Candida as the commensal flora plays role to maintaining balance microbiota component and maintaining the local immune system. When the tolerance mechanism is disturbed, the yeast will undergo transition to hyphae.21 Some virulence factors of candida are proteolytic enzymes, phospholipase, and toxins. ${ }^{4}$ In the form of hyphae, a biofilm layer is formed that adhere strongly and invade the outer layer of vaginal epithelium. ${ }^{21}$ Biofilms are formed in four phases: the initial phase of attachment, which is when yeasts adhere to the surface (first 1-3 hours); intermediate phase of biofilm formation (11-14 hours); polymeric matrix (PEM) maturation phase which will enhance the penetration of all cell layers (20-48 hours); and biofilm spread over the cell surface (> 24 hours). ${ }^{11}$

Secretory aspartyl proteinases, the stability factors in epithelial cells, are proteolytic enzymes that degrade tissue barrier proteins, such as E-cadherin. Secretory aspartyl proteinases also have the ability to degrade natural immunity (e.g. complement) and acquired immunity (e.g. antibody). This function will cause candida to develop. In women with vulvovaginal candidiasis and recurrent vulvovaginal candidiasis, there is a high level of active SAP enzymes, compared to asymptomatic women.21 SAP gene expression is influenced by several factors including environmental $\mathrm{pH}$ and the form of candida. ${ }^{21}$ Phospholipase enzymes will target the phospholipid membrane. Phospholipase will destroy phospholipid components, initiate cell lysis and facilitate the penetration of epithelial cell by the candida.11 Mycotoxins, one of which is gliotoxin, will inhibit phagocyte activity or suppress the local immune system. ${ }^{4}$

\section{Clinical manifestations}

The main complaints from patient are severe pruritus, irritation, abnormal vaginal discharge. The abnormal vaginal discharge is mucoid or liquid with cottage chesses. Patients also complain dyspareunia or painful urination. 1

During physical examination, the vulva is erythema, edema with fissure, erosion and ulcerated, but also can be seen without any physical changes. Pseudo membrane with thrush and matted mycelia, leucocytes and epithelial cells can be found in vaginal walls. ${ }^{1}$ Vulvovaginal candidiasis will impact the patient's quality of life and sexual activities. These can be resulted in depression and anxiety. 3,5 Female patients 
with recurrent vulvovaginal candidiasis have lower selfconfidence, depression, and less satisfied with their self. About $53 \%$ of female with recurrent vulvovaginal candidiasis also diagnosed with depression or anxiety. ${ }^{3}$

\section{Diagnosis}

Recurrent vulvovaginal candidiasis is vulvovaginal candidiasis that recurs for 4 times or more in a year. ${ }^{1}$ Anamnesis, physical examination and microscopic examination will help to diagnose recurrent vulvovaginal candidiasis. ${ }^{4,9}$ Microscopic examination can be done with physiological saline, $10 \%$ potassium hydroxide $(\mathrm{KOH})$, or Gram staining from vaginal discharge or pseudomembrane to look for budding yeast, pseudohyphae or hyphae.1,23

Some findings in microscopic examination are gram positive candida, abundant pseudohyphaes but budding yeasts are rarely to be found. The most common pathogen that cause recurrent vulvovaginal candidiasis is candida albicans. In vitro examination shows many pseudohyphaes that can be detected with gram staining from vaginal discharge. ${ }^{1}$ Microscopic examination with physiological saline and $10 \% \mathrm{KOH}$ has sensitivities about $40-70 \% .5$ The vaginal with recurrent vulvovaginal candidiasis mostly has normal $\mathrm{pH}$ (4-4.5), however, the increase of $\mathrm{pH}$ should be considered as other infection such as bacterial vaginosis, trichomoniasis, or multiple infections. 4,5,9

Colpocytology examination with Papanicolau technic will able to show pseudohyphae/ pseudomiselium and blastospores. ${ }^{22}$ Homology DNA examination will show accurate result within a few hours. 5 Other examination to diagnose recurrent vulvovaginal candidiasis is Polymerase Chain Reaction (PCR) to detect specific species of Candida. ${ }^{5}$ About $55 \%$ cases of positive culture of fungi will have negative microscopic examination result. ${ }^{4}$ The best medium for symptomatic cases with normal $\mathrm{pH}$ and negative microscopic result is Sabouraud agar (Figure 2). 4,21

The aim of fungal culture is to determine the specific species as the etiology of each case. ${ }^{1}$ The culture should be followed with microbiological examination to determine the sensitivity of azole agents to candida sp. 5

\section{Treatment}

Recurrent vulvovaginal candidiasis is a multifactorial disease. The first priority of treatment is to eliminate predisposition factors. Fungal culture has to be done to confirm the diagnosis and to identify specific species of Candida before began the therapy. ${ }^{4}$ Antifungal, both topical or systemic, should be given to all recurrent vulvovaginal candidiasis cases until the patient become asymptomatic and the culture is negative. 4

There are some species candida that can cause recurrent vulvovaginal candidiasis. Recurrent vulvovaginal candidiasis which caused by Candida albicans, Candida tropicalis, Candida parapsilosis should be treated with fluconazole. The initial dose of fluconazole is $150 \mathrm{mg}$ every 3 day for 3 doses (first, fourth and seventh day). The maintenance dose of fluconazole is $150 \mathrm{mg}$ in every week for six months. Other treatment of choice is $200 \mathrm{mg}$ itraconazole twice a day for 3 consecutive days, followed by 100-200 mg of itraconazole once daily for six months. Topical antifungal should be prescribed to the patient. Some topical antifungal of choice are clotrimazole intravaginal cream $1 \%$ for 7 days; clotrimazole intravaginal cream $2 \%$ for 3 days; miconazole intravaginal cream $2 \%$ for 7 days; miconazole intravaginal cream $4 \%$ for 3 days; tioconazole ointment $6.5 \%$ single dose; terconazole intravaginal cream $0.4 \%$ for 7 days; terconazole intravaginal cream $0,8 \%$ for 3 days; terconazole $80 \mathrm{mg}$ intravaginal suppositories for 3 days; butoconazole intravaginal cream $2 \%$ single dose followed by maintenance miconazole $1200 \mathrm{mg}$ intravaginal suppositories every week for six months. ${ }^{4}$

For recurrent vulvovaginal candidiasis that caused by Candida glabrata should be treated with boric acid $600 \mathrm{mg}$ intravaginal suppositories once a day for 14 days or nystatin intravaginal suppositories $100.000 \mathrm{U}$ for 14 days. Both can be used as maintenance therapy with the same dose as the initial treatment. ${ }^{5}$ Long term effects of boric acid is still need to be confirmed, however, boric acid should not be used during pregnancy. 4 For VCR which is caused by Candida krusei can be treated with all modalities mentioned 
before, but fluconazole.

In case of azole-resistant Candida species, diagnosis process should include antimicrobial resistance in vitro examination to determine the minimal inhibitory concentration (MIC). Some modalities for azoleresistant Candida species are boric acid $600 \mathrm{mg}$ intravaginal suppositories once a day for 14 days; nystatin 100.000 IU intravaginal tablet suppositories for 14 days; amphotericin intravaginal cream or tablet suppositories 5-10\% once daily for 14 days; flucytosine intravaginal cream $17 \%$ for 14 days or combination of Amphotericin B with flucytosine. 5 In HIV patients with recurrent vulvovaginal candidiasis, boric acid $600 \mathrm{mg}$ intravaginal suppositories capsule for 14 days should be given as treatment. ${ }^{18}$
In a small size study, they found that sugar restriction for recurrent vulvovaginal candidiasis made a clinical improvement. Addition of antihistamine also reduce the pruritus. Another alternative for patient that allergic to azole antifungal agent is systemic hyposensitization with candida's antigen give in cutaneous access. ${ }^{4}$ In the case of recurrent vulvovaginal candidiasis that presents after the use of antibiotic, $150 \mathrm{mg}$ of fluconazole is given before antibiotics were given and every 3-4 days, as prophylactic agent. Depression or anxiety frequently occur with recurrent vulvovaginal candidiasis, so psychotherapy agents can be given twice a week during the treatment. 3 


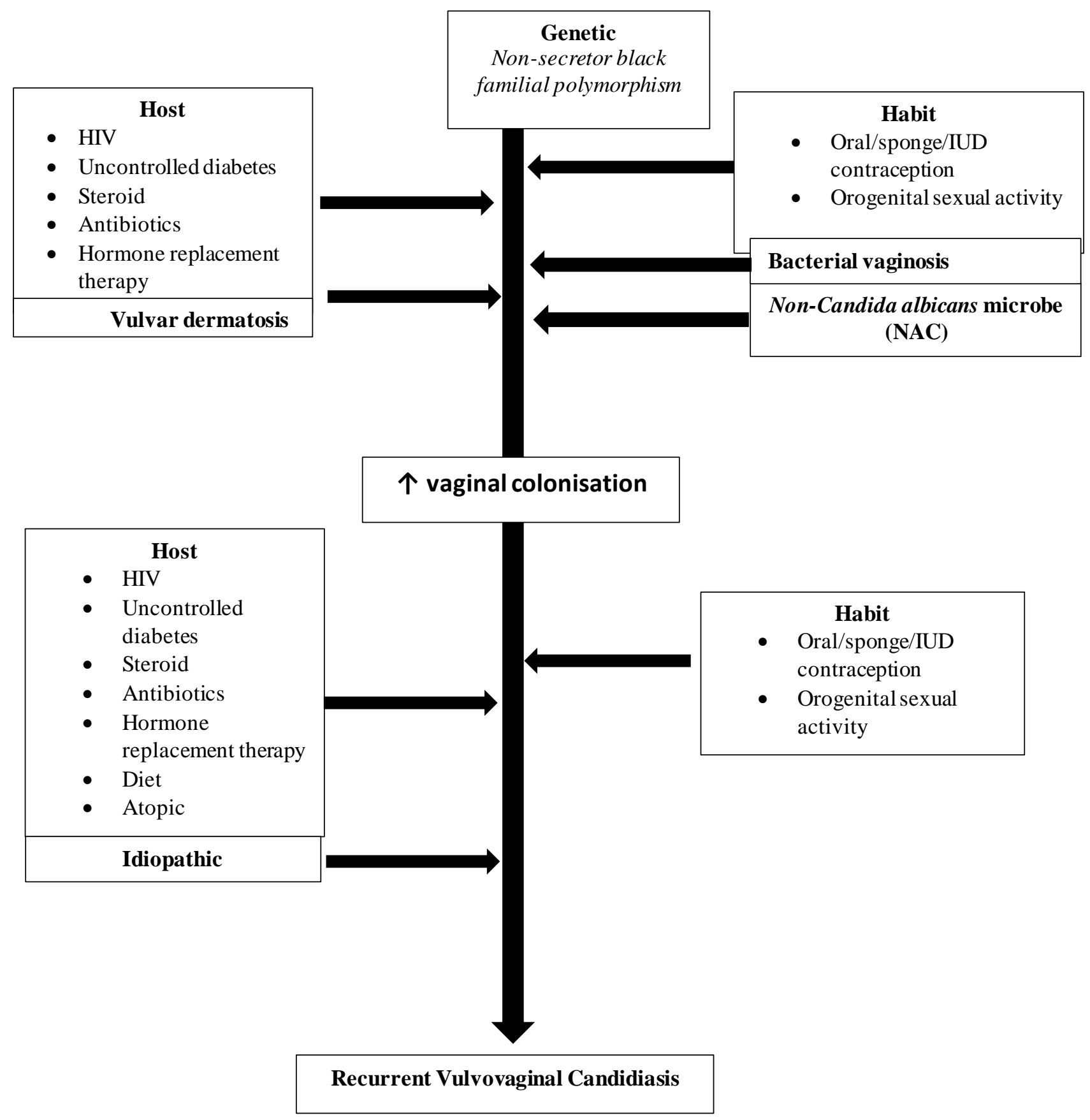

Figure 1. Predisposition factors of vulvovaginal candidiasis and recurrent vulvovaginal candidiasis 5 


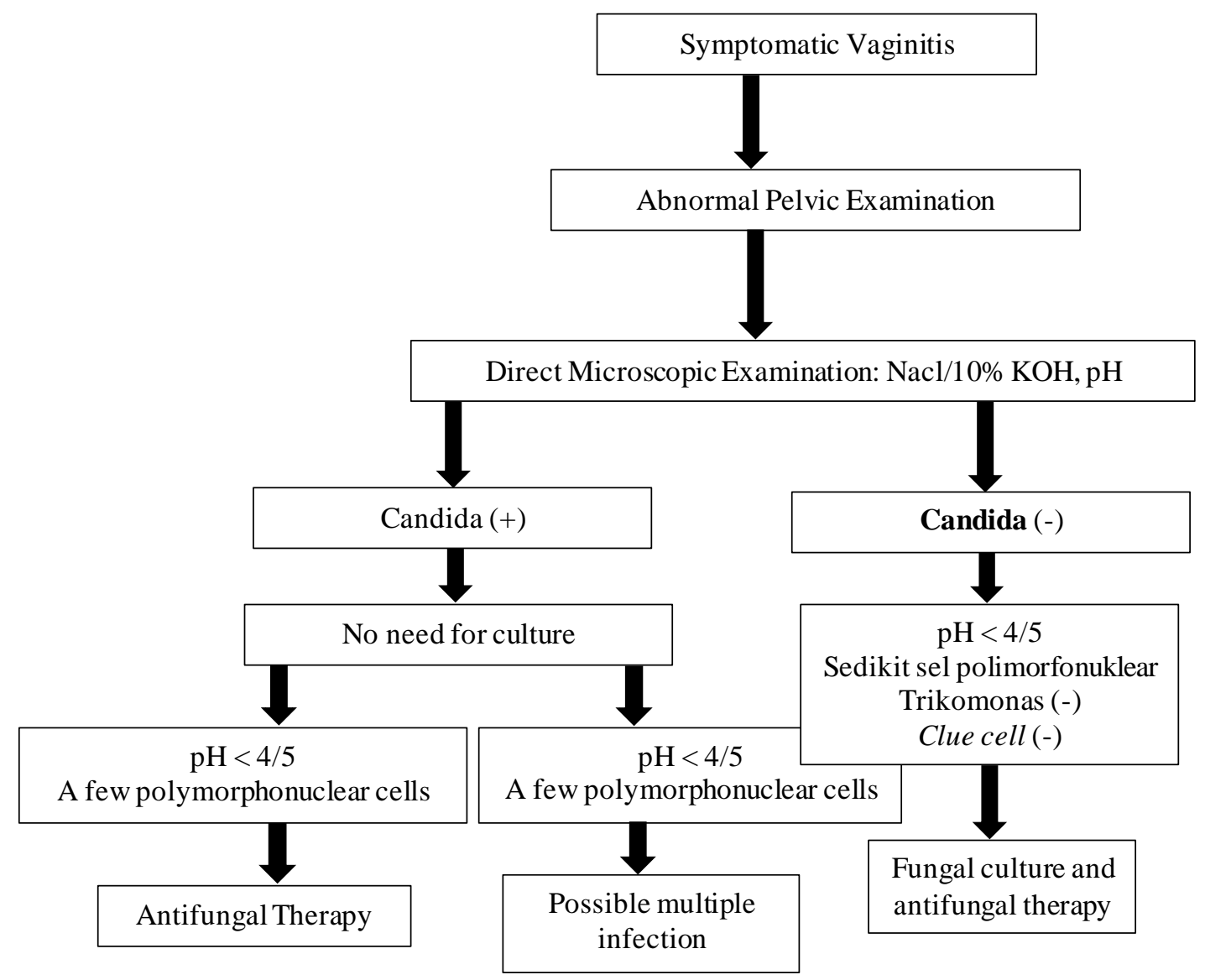

Figure 2. Diagnostic and Therapy Algorithm of Recurrent Vulvovaginal Candidiasis 4

\section{Conclusion}

The incidence of recurrent vulvovaginal candidiasis is high in female and related to various factors such as genetic, host, habit, idiopathic and the candida itself. Recurrent vulvovaginal candidiasis can cause variety effects such as decreased quality of life, depression, and sexual dysfunction. Diagnosis recurrent vulvovaginal candidiasis is based on anamnesis, physical examination, microscopic examination and fungal culture. The purpose of recurrent vulvovaginal candidiasis treatment is to eliminate the predisposition factors of the host and to give the right regiment based on culture and microbial resistance test.

\section{References}

1. Pudjiati SR, Soedarmadi. Kandidosis Genitalis. In: Djuanda A. Ilmu Penyaki Kulit dan
Kelamin. $7^{\text {th }}$ ed. Jakarta-Balai Penerbit FKUI; 2016. p.171-9.

2. Rosati D, Bruno M, Jaeger M, Oever JT, Netea MG. Recurrent vulvovaginal candidiasis: an immunological perspective. Microorganism. 2020; 8:1-15.

3. Blostein F, Sparenberg EL, Wagner J, Foxman B. Recurrent vulvovaginal candidiasis. Ann Epidemiol. 2017; 27:575-82.

4. Sobel JD. Genital candidiasis. In: Gross G, Tyring SK. Sexually transmitted infections and sexually transmitted diseases. Berlin: Springer International Publishing AG; 2011. p. 613-24.

5. Sobel JD. Recurrent vulvovaginal candidiasis. Am J Obstet Gynecol. 2016:15-21

6. Nevodic B, Posteraro B, Leoncini E, et al. Mannose-binding lectin codon 54 gene polymorphism and vulvovaginal candidiasis: a 
systematic review and meta-analysis. Biomed Res Int. 2014:0;1-7

7. Rosentul DC, Delsing CE, Jaeger M, Plantinga TS, Oosting $\mathrm{M}$, Costantini $\mathrm{I}$, et al. Gene polymorphisms in pattern recognition receptors and susceptibility to idiopathic recurrent vulvovaginal candidiasis. Front Microbiol. 2014;5:483.

8. Jaeger M, Carvalho A, Cunha C, Plantinga TS, van de Veerdonk F, Puccetti M, et al. Association of a variable number tandem repeat in the NLRP3 gene in women with susceptibility to RVVC. Eur $J$ Clin Microbiol Infect Dis. 2016;35:797e801.

9. Sobel JD.Vulvovaginal candidiasis. In: Holmes KK, Sparling PF, Stamm WE, Piot P, Wasserheit JN, Corey L, et al. Sexually transmitted disease. 4th edition. New York: McGraw-Hill; 2008. p. 823-838.

10. Aguin TJ, Sobel JD. Vulvovaginal candidiasis in pregnancy. Curr Infect Dis Rep. 2015;17(6):1520.

11. Rodrigues CF, Rodrigues ME, Henriques M. Candida sp. Infections in patients with diabetes mellitus. J Clin Med. 2019; 8:1-41.

12. Smith SB, Ravel J. The vaginal microbiota, host defence and reproductive physiology. J Physiol. 2017;595(2):451-63.

13. Amabebe E, Anumba DOC. The Vaginal microenvironment: the physiologic role of lactobacilli. Front Med. 2018;5:1-11.

14. Zinkeviciene A, Vaiciulioniene N, Baranauskiene I, Kvedariene V, Emuzyte R, et al. Cutaneus yeast microflora in patients with atopic dermatitis. Cent Eur J Med. 2011;6(6):713-19.

15. Talaei Z, Sheikhbahaei S, Ostadi V, Hakemi MG, Meidani M, Nangshineh E, et al. Recurrent vulvovaginal candidiasis: could it be related to cell-mediated immunity defect in response to candida antigen. 2017;11(3):134-41.

16. Neves NA, Carvalho LP, Oliveira MAMD, Giraldo PC, Bacellar O, Cruz AA, et al. Association between atopy and recurrent vaginal candidiasis. Clin Exp Immunol. 2005; 142:16771.

17. Farhan MA, Moharram AM, Salah T, Shaaban OM. Types of yeast that cause vulvovaginal candidiasis in chronic users of corticosteroids. Med Mycol J. 2018;0:1-7.

18. Pudjiati SR, Ramali LM. Kandidiasis mukokutan pada pasien HIV dan AIDS. In: Hidayati AN, Daili $\mathrm{SF}$, Niode $\mathrm{NJ}$, Indriatmi W, Budiono $\mathrm{SE}$, Barakbah J. Manifestasi dan tatalaksana kelainan kulit dan kelamin pada pasien HIV/AIDS. $1^{\text {st }}$ ed. Jakarta-Balai Penerbit FKUI; 2018. p.135-51.

19. Stockdale CK, Boardman L. Diagnosis and treatment of vulvar dermatoses. Obstet Gynecol. 2018;0:1-16.

20. Vij A, Sood A, Piliang M, Mesinkovska NA. Infection or allergy? The multifaceted nature of vulvar dermatoses. Int $\mathrm{J}$ Womens Dermatol. 2015;0:170-2.

21. Cassone A. Vulvovaginal Candida albicans infections: pathogenesis, immunity and vaccine prospects. BJOG-Int J Obstet. 2015;122:78594.

22. Passos MRL. Atlas of sexually transmitted diseases clinical aspects and differential diagnosis. Switzerland: Springer International Publishing AG; 2018. p. 203-37.

23. Workowski KA, Bolan GA. Centers for Disease Control and Prevention. Sexually transmitted diseases treatment guidelines, 2015. MMWR Recomm Rep. 2015;64(3): p. 1-137. 